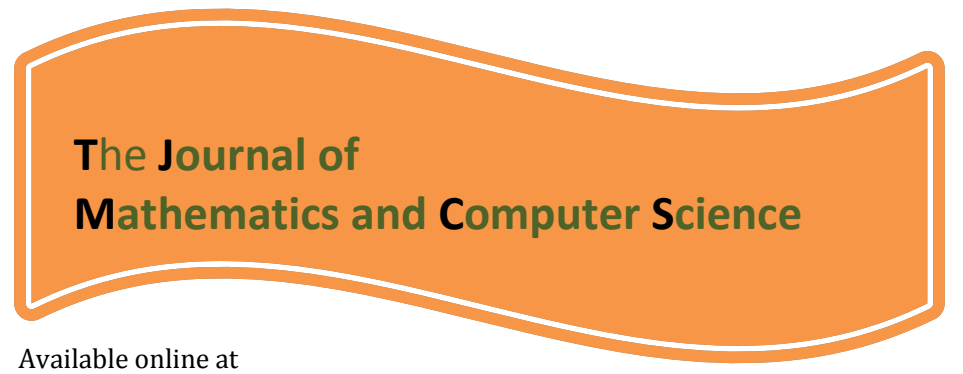

http://www.TIMCS.com

The Journal of Mathematics and Computer Science Vol .3 No.3 (2011) 329 - 338

\title{
A Priority Scheduler Based Qos for Dynamic Source Routing Protocol Using Fuzzy Logic in Mobile Ad-Hoc Network \\ Saber Ghasempour ${ }^{1}$,Seyed Hossein Kamali², Maysam Hedayati ${ }^{3}$, Reza Shakerian ${ }^{4}$
}

\author{
${ }^{1}$ Department of Mathematics, Payame Noor University,PO BOX 19395-3697 ,Tehran , Iran \\ ${ }^{2}$ Islamic Azad University of Qazvin Branch, Qazvin, Iran \\ 3 Islamic Azad University-Ghaemshahr Branch, Ghaemshahr, Iran \\ 4 Payame Noor University,PO BOX 19395-3697 ,Tehran , Iran
}

Received: August 2011, Revised: November 2011

Online Publication: December 2011

\begin{abstract}
Mobile ad hoc network is an autonomous system of mobile nodes characterized by wireless links. A wireless Ad-hoc network consists of wireless nodes communicating without the need for a centralized administration, in which all nodes potentially contribute to the routing process.

In this paper we design a fuzzy-based priority scheduler to determine the priority of the packets. We analyze packet scheduling algorithm to find those that most improve performance in congested network. Hence, a scheduling algorithm to schedule the packet based on their priorities will improve the performance of the network. Packet schedulers in wireless ad hoc networks serve data packets in FIFO order. Here, we present a fuzzy based priority scheduler for mobile adhoc networks, to determine the priority of the packets using Destination Sequenced Distance Vector (DSRs) as the routing protocols. The performance of this scheduler has been studied using OPNET simulator and measured such as packet delivery ratio, end-to-end delay and throughput. It is found that the scheduler provides overall improvement in the performance of the system when evaluated under different load and mobility conditions.
\end{abstract}

Keywords: Scheduling Algorithms; Fuzzy Logic; DSR; OPNET; MANET. 


\section{S. Ghasempour, S.H. Kamali, M. Hedayati, R. Shakerian/ TJMCS Vol .3 No.3 (2011) 329 - 338}

\section{INTRODUCTION}

Ad hoc network is a collection of wireless nodes, which form a temporary network without relying on the existing network infrastructure or centralized administration. Ad hoc networks form a multihop network, where the communication is over the wireless channel, hopping over several mobile nodes. In recent years, a number of unicast routing protocols have been proposed. Routing and packets forwarding in ad hoc networks is a fairly unexplored area. In today's network, data transmission between multiple senders and receivers is becoming increasingly important. There are many applications which send from a single source to multiple destinations or from multiple senders to multiple receivers. In addition, it also provides a simple and robust communication mechanism when the receiver's individual addresses are unknown or changeable. It also can improve the utilization of the wireless link, when sending multiple copies of messages and exploit the inherent broadcast property of wireless transmission. Hence, multicasting plays an important role in ad hoc networks $[1,2,3,4]$.

A collection of nodes that communicate with each other by forming a multihop radio network and maintaining connectivity in a decentralized manner is called an ad hoc network. In the proposed networks as shown in Fig.1, the mobility of nodes and the error prone nature of the wireless medium pose many challenges like frequent route changes and packet losses. Such problem increases packet delay and decrease throughput. To improve the performance and maintain the QoS in MANET the scheduler can be used. C. Gomathy et al. [5] has been design a fuzzy based priority scheduler to determine the priority of the packets. Kumar et al. [6] defined how to improve the end-to-end QoS target in MANET. Mary Bader et al. [7] has focused primarily on routing protocols -how to router packet hop by hop as efficient as possible and medium access control (MAC - how to share the medium efficiently. Based on those papers, we are trying to explore how to achieve the better performance of end-to-end QoS target in MANET.

QoS routing algorithms for wired networks relies on the availability of the precise state information. However, nature of wireless channel makes the available state information inherently imprecise. Moreover, the topology is fluid; nodes may join, leave and rejoin the network at any time at any location. The QoS routing problem is to find a route that has sufficient resources to satisfy the QoS requirements. This requires the translation of the user requirements into independent network parameters, determination of the resources, the discovery of the optimal route with sufficient resources, resource reservation along the route and maintenance of the route. The QoS constraints that need to be met are delay, bit error rate, bandwidth, route length etc. along with MANET specific requirements like energy, route stability and route reliability. Once the resources are estimated, a route discovery protocol is run to determine the feasible routes [8]. 


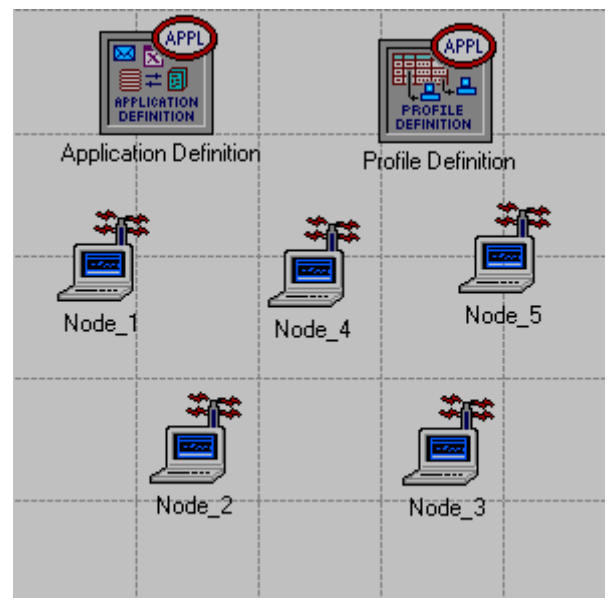

Figure 1. A proposed model of ad hoc network.

In this paper, to provide a fuzzy based priority scheduling scheme, this improves the QoS parameters in wireless ad hoc network. Hence, introducing a scheduling algorithm to determine which queued packet to process next will improve the overall end-to-end performance. Without scheduling, the packets will be processed in FIFO (First in First out) manner and hence there is frequently dropped the packets. A scheduler should schedule the packets to reach the destination quickly, which are at the verge of expiry. To incorporate the scheduler for the existing routing protocols, various routing protocols are studied $[9,10,11]$.

\section{Scheduling AlgorithmS}

Ad hoc networks have several features that may produce unique queuing dynamics. The choice of scheduling algorithm has a significant effect on the overall end-to-end performance when traffic load is high. This motivated us to evaluate the existing scheduling algorithms and propose a new fuzzy-based scheduler. The effects of setting priorities to control and data traffic are studied. The study is performed with the three multicast protocols as described in the previous section.

There are several scheduling policies for different network scenarios. Different routing protocols use different methods of scheduling. The drop-tail policy is used as a queue management algorithm in all scheduling algorithms for buffer management. For the scheduling algorithms that give high priority to control packets, different drop policies are used for data and control packets when the buffer is full. When the incoming packet is a data packet, the data packet is dropped. When the incoming packet is a control packet, the last enquired data packet is dropped. If queued packets are control packets, the incoming control packet is dropped. Except for the no-priority scheduling algorithm, all the other scheduling algorithms give higher priority to control packets than to data packets. The differences in the algorithms are in assigning priority between data packets. In no-priority scheduling, both control and data packets are served in FIFO order. In the priority scheduling, control and data packets are maintained in separate queues in FIFO order and high priority is assigned to control packets. Currently, only this scheme is used in mobile ad hoc networks [12]. 
When looking onto the effect of setting priorities to data packets and considering the suitability of the different types of scheduling algorithms for MANET, several scheduling schemes were studied in literature. In order to consider the effect of setting priorities to data packets, these schedulers give high priority to control packets. Their differences are in assigning priorities among data queues. Fig. 2 shows the priority scheduler for data packets. Weighted-hop and weighteddistance scheduling methods use the distance metrics. Weighted-hop scheduling gives higher weight to data packets that have fewer remaining hops to traverse. If the packet has fewer remaining hops, then it has to reach the destination quickly. The data packets can be stored in round robin fashion. The remaining hops to traverse can be obtained from packet headers. Weighted-distance scheduling gives higher weight to data packets which have shorter geographic distances. The remaining distance is the distance between a chosen next hop and a destination. Round-robin scheduling maintains per-flow queues. The flow can be identified by a source and destination pair. Here each flow queue is allowed to send one packet at a time in a round-robin fashion. In the greedy scheduling scheme, each node sends its own data packets before forwarding those of other nodes [7]. The data packets of other nodes are serviced in FIFO order.

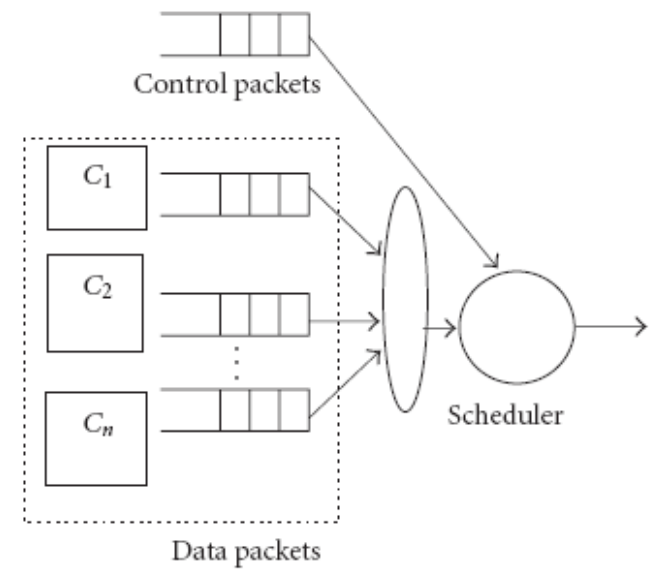

Figure 2. Priority scheduler for data packets.

Two other schedulers are the earliest deadline first (EDF) and the virtual clock (VC) [2]. In EDF, a packet arriving at time $t$ and having delay bound $d$ has a deadline $t+d$. The packets will be scheduled based on this deadline. In $\mathrm{VC}$, a packet with size $L$ of a flow, with rate $r$, has a priority index $L / r$ plus the maximum of current time $t$ and priority index of the flow's previous packet. In these scheduling algorithms, the parameters used to find the priority of data packets are remaining hops to traverse, distance, per-flow queues, and greediness of nodes, delay bound, and flow rate. With the thorough study of ad hoc networks, and the above-mentioned scheduling algorithms, it is found that a number of metrics can be combined into a single decision so as to find the crisp value of the priority of packets. Our solution to determine the priority index of the packets utilizes the fuzzy logic concept $[1,7]$. The three input variables, namely, expiry time of packet, queue length of the node, and Data rate of the source, are considered and the application of fuzzy logic to combine these variables and hence find the priority index of the packet is found to be suitable. This led to the design of a fuzzy-based priority scheduler. 


\section{THE FUZZY SCHEDULER}

\section{A. Fuzzy Scheduler}

The fuzzy scheduler proposed here, calculates the priority index of each packet. The fuzzy scheduler uses two input variables and one output variable. The two input variables to be fuzzified are the data rate and channel capacity of the nodes to which the packet is associated with. The inputs are fuzzified, implicated, aggregated and defuzzified to get the crisp value of the output. The linguistic variables associated with the input variables are Low (L), medium (M) and high (H). For the output variable, priority index, six linguistic variables are used. They are, very low (VL), low (L), medium $(\mathrm{M})$, average $(\mathrm{A})$, high $(\mathrm{H})$ and very high $(\mathrm{VH})$. The Table 1 shows the fuzzy conditional rules for the fuzzy scheduler.

TABLE I. FUZZY RULE BASE

DR-DATA RATE, CC-CHANNEL CAPACITY

\begin{tabular}{|c|c|c|c|}
\hline CC & L & M & H \\
\hline L & L & A & M \\
\hline M & VL & M & H \\
\hline H & A & A & VH \\
\hline
\end{tabular}

The first rule can be interpreted as, "If (channel capacity is low) and (data rate is low) then priority index is low". Since in this rule, data rate and channel capacity are low and the priority index is set to be low. Similarly the other rules have been developed. The output priority index, if very low, indicates that packets are associated with low delay, are attached with a very high priority and should be immediately scheduled. The surface viewer for the fuzzy scheduler in case of constant data rate and channel capacity is shown in Fig. 3 Similarly, if the priority index is very high, it indicates that packets are attached with least priority and will be scheduled only after the high priority packets are scheduled [7].

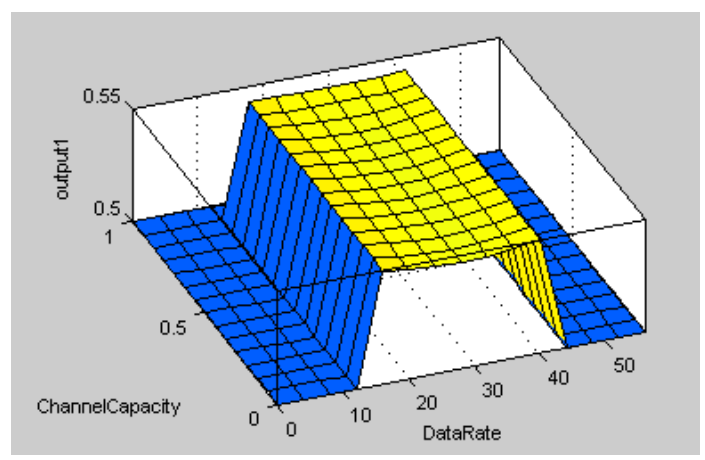

Figure 3. Surface viewer for the fuzzy scheduler in case of constant data rate and channel capacity. 


\section{B. Fuzzy Logic}

Fuzzy logic implements human experiences and preferences via membership functions and fuzzy rules. The application of fuzzy logic to problems of traffic control in networks is more attractive. Since it is difficult for a network to acquire complete statistics of the input traffic, it has to make a decision based on incomplete information. Hence the decision process is full of uncertainty. It is advantageous to use the fuzzy logic in the target system because it is flexible and capable of operating with imprecise data. Basically the fuzzy system consists of four blocks, namely, fuzzifier, defuzzifier, inference engine, and fuzzy knowledge base [4]. The following section explains the working of the general fuzzy system.

\section{1) Fuzzification Of Inputs And Outputs}

The first step is to take the inputs and determine the degree to which they belong to each of the appropriate fuzzy sets via membership functions. The input is always a crisp numerical value limited to the universe of discourse of the input variable and the output is a fuzzy degree of membership in the qualifying linguistic set (always the interval between 0 and 1). A fuzzy set $A$ in the universe of discourse $U$ is a set of ordered pairs $\left\{\left(x_{1}, \mu_{A}\left(x_{1}\right)\right),\left(x_{2}, \mu_{A}\left(x_{2}\right)\right), \ldots,\left(x_{n}, \mu_{A}\left(x_{n}\right)\right)\right\}$, where $\mu_{A}: U \rightarrow[0,1]$ is the membership function of the fuzzy set $A$ and $\mu_{A}\left(x_{i}\right)$ indicates the membership degree of $x_{i}$ in the fuzzy set $A$.

\section{2) Fuzzy Inference Process}

If a fuzzy system has $n$ inputs and a single output, its fuzzy rules $R_{j}$ can be of the following general format. ( $\left.R_{j}\right)$ If $X_{1}$ is $A_{1 j}, X_{2}$ is $A_{2 j}, X_{3}$ is $A_{3 j}, \ldots$, and $X_{m}$ is $A_{m j}$, then $Y$ is $B_{j}$. The variables $X_{i}\{i=1,2,3, \ldots, n\}$ appearing in the antecedent part of the fuzzy rules $R_{j}$ are called the input linguistic variables, the variable $Y$ in the consequent part of the fuzzy rules $R_{j}$ is called the output linguistic variable. The fuzzy sets $A_{i j}$ are called the input fuzzy sets of the input linguistic variable $X_{i}$ and the fuzzy sets $B_{j}$ are called the output fuzzy sets of the output linguistic variable $Y$ of the fuzzy rules $R_{j}$.

\section{3) Implication Method}

Before applying the implication method, the rule's weight must be taken care of. Every rule has a weight (a number between 0 and 1 ), which is applied to the number given by the antecedent. Once proper weighting has been assigned to each rule, the implication method is implemented. A consequent is a fuzzy set represented by a membership function, which weighs appropriately the linguistic characteristics that are attributed to it. The consequent is reshaped using a function associated with the antecedent (a single number) $[1,12]$. The input for the implication process is a single number given by the antecedent, and the output is a membership function, implemented for each rule.

\section{Performance Evaluation}

\section{A. Performance Metrics}

The following metrics are used to evaluate the effect of the modified fuzzy scheduler. 


\section{S. Ghasempour,S.H. Kamali, M. Hedayati, R. Shakerian/ TJMCS Vol .3 No.3 (2011) 329 - 338}

(i) Packet Delivery Ratio: Packet delivery ratio is the ratio of the number of data packets actually delivered to the destinations to the number of data packets supposed to be received. This number presents the effectiveness of the protocol.

(ii) Average End-To-End Delay: This indicates the end-to-end delay experienced by packets from source to destination. This includes the route discovery time, the queuing delay at node, the retransmission delay at the MAC layer, and the propagation and transfer time in the wireless channel [2].

\section{B. Simulation and Performance evaluation using OPNET}

Our simulation modeled a network of mobile nodes placed randomly within $250 \times 250$ meter area. Each simulation is run for 600 seconds of simulation time. Multiple simulations run with different seed values were conducted for each scenario and collected data was averaged over those simulated results. A free space propagation model was used in our simulation. Each source transmits data packets at a minimum rate of 5 packets/sec. and maximum rate of 15 packets/sec. The traffic load is varied, by changing the number of data and the effect is evaluated on scheduler with DSR routing protocols. The priority index that has to be associated with each packet is determined using fuzzy logic. The inputs to determine the priority index are the channel capacity of the node in which the packet is present and the data rate. The inputs are received for a network and rules are evaluated using these inputs. Each evoked rule provides an output membership function. These output membership functions are then implicated, aggregated and the crisp priority index is calculated from this aggregated curve using centric method of defuzzification. As a result of this, the number of packets delivered to the sender, the end-to-end delay of the packet transmission and the throughput improves. The input channel capacity is fixed, which is present in the physical layer of the simulator. For each hop it reduces by 1 if the channel capacity is full and packet suffers excessive and its falls to zero. As a result of this, the packet is dropped. If this variable is used as input to the scheduler for finding the priority index, a packet with a very low value is given the highest priority. Hence due to this, the dropping of packets experience multihops gets minimized. The next input to the scheduler is the data rate of transmission and it is normalized. If the packet is present in a highly crowded node, it suffers excessive delays and gets lost. So, such a packet is given a higher priority and hence it gets saved. The priority index is calculated with the inputs obtained from the network layer. This is then added to the header associated with the packet [6]. Hence whenever the packet reaches a node, its priority index is calculated and it is attached with it. Each node has three queues. Each queue in the node is sorted based on the priority index and the packet with the lowest priority index (i.e. packets with the highest priority), is scheduled next, when the node gets the opportunity to send. By this method of scheduling, the overall performance increases. The performance of the network with the fuzzy code and without the code is studied under various conditions such as variation in network load and mobility of the nodes. In this simulation, the mode mobility is set at $5 \mathrm{~m} / \mathrm{s}$ and network traffic load is large. The routing protocol is chosen to be DSR. Now the impact of node density on scheduler performance has been evaluated. The packet delivery ratio as a function of number of nodes is 
shown in Fig. 4. The performance of fuzzy scheduler with reference to the packet delivery ratio is much improved as compared with that of one without scheduler. It is also seen that for small loads, the scheduler does not provide much improvement, but the traffic load is increased the improvement is more.

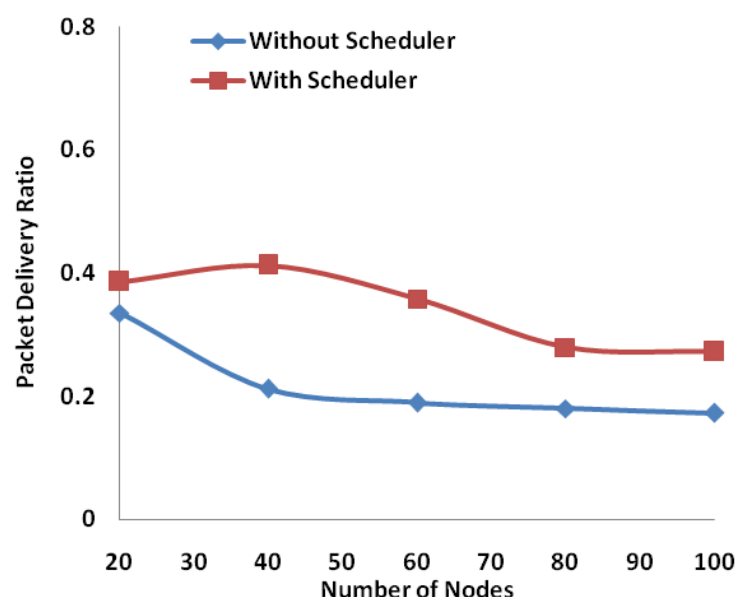

Figure 4. Packet delivery ratio as a function of network size.

When nodes reach the simulation boundary, they are bounced back and continue to move. The mode movement speed or the mobility of the nodes is varied from $0 \mathrm{~m} / \mathrm{s}$ to $20 \mathrm{~m} / \mathrm{s}$. Number of nodes is set as five and the routing protocol is selected to be DSR. The above simulation scenario, the fuzzy scheduler is tested with DSR routing protocols available in OPNET simulator. The placement of node is set to random and number of nodes is selected as five. The simulation is run with number of packets to be transmitted equal to 80000 packets. The performance is evaluated with different seed value and the average value is taken for all the performance metrics. From the simulated results, the packet delivered for DSR improves by 39\% for a total transmission of 80000 packets. The end-to-end delay decreases by around 0.35seconds for DSR. It's shown in Fig. 5. From the results it is clear that the fuzzy scheduler works better for the DSR protocols, tested under different load and mobility conditions.

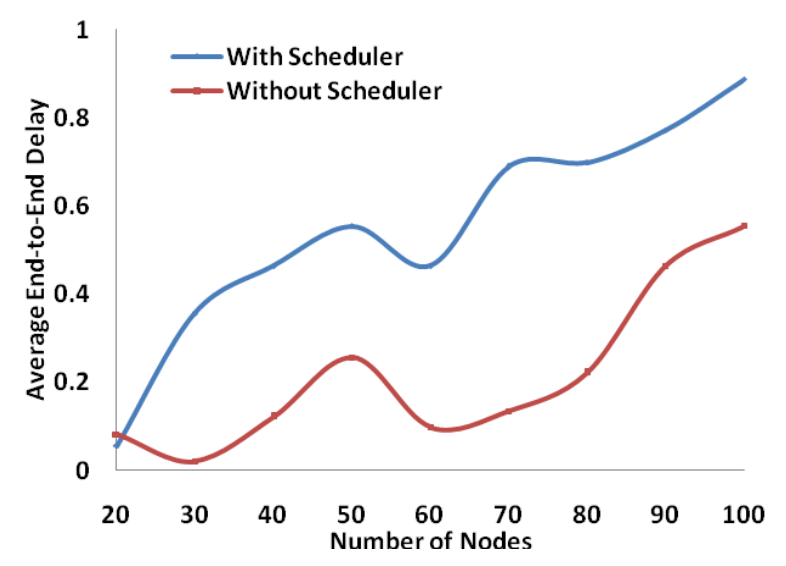

Figure 5. Average end-to-end delay Vs network size. 


\section{S. Ghasempour,S.H. Kamali, M. Hedayati, R. Shakerian/ TJMCS Vol .3 No.3 (2011) 329 - 338}

\section{ConClusion}

In this paper, the performance of the fuzzy scheduler has been proposed for wireless ad hoc network using OPNET simulator. It is found from the results that, priority scheduling helps in effective routing of packets without minimum loss and delay. In this proposed model as shown in fig.1, from the simulation results, the packet delivered for DSR improves by $39 \%$ for a total transmission of packets and end-to-end delay decreases by around 0.35 seconds.also, to provide a fuzzy based priority scheduling scheme, this improves the QoS parameters in wireless ad hoc network. It combines the input parameters such as channel capacity and data rate and to find the priority index. The crisp value is calculated based on input of fuzzy scheduler, which is derived from the network. The memberships functions and rule bases of the fuzzy scheduler has been designed. The coding is done in C language and output is verified using MATLAB fuzzy tool. We have addressed a fuzzy-based priority scheduler for data packets, which improves the quality-ofservice parameters in mobile ad hoc networks. The fuzzy scheduler attaches a priority index to each packet in the queue of the node. Unlike the normal sorting procedure for scheduling packet, a crisp priority index is calculated based on the inputs such as queue length, data rate, and expiry time of packets, which are derived from the network. The membership functions and rule bases of the fuzzy scheduler are carefully designed.

\section{REFERENCE}

[1] C. Gomathy and S. Shanmugavel, "Implementation of modified Fuzzy Priority Scheduler for MANET and performance analysis with mixed traffic," in Proc. 11th National Conference on Communication (NCC '05), Indian Institute of Technology, Kharagpur, India, January 2005.

[2] V. Kanodia, C. Li, A. Sabharwal, B. Sadeghi, and E. Knightly, "Distributed priority scheduling and medium access in ad hoc networks, "Wireless Networks, vol. 8, no. 5, pp. 455-466, 2002.

[3] S. Radha and S. Shanmugavel, "Multicasting in ad hoc networks using NTP protocol," in Proc. 15th International Conference on Computer and Communication (ICCC '02), pp. 144-160, Bandra, Mumbai, India, August 2002.

[4] C. Gomathy and S. Shanmugavel, "Fuzzy based Priority Scheduler for mobile ad hoc networks," in Proc. 3rd International Conference on Networking (ICN '04), Gosier, Guadeloupe, French Caribbean, February-March 2004.

[5] C. Gomathy and S. Shanmugavel "Supporting QoS in MANET by Fuzzy Priority Scheduler and Performance Analysis with Multicast Routing Protocols" EURASIP Journal on Wireless Communication and Networking 2005:3, 426-436.

[6] Kumar Manoj, S.C. Sharma, Sandip Vijay and Amit Dixit "Performances Analysis of Wireless Adhoc Network Using OPNET Simulator "International Conference on "Intelligent Systems and Networks” (ISN-08) ISTK Haryana, 22-24th 2008, 267-270 Feb. 2008. 
[7] B. G. Chun and M. Baker, "Evaluation of packet scheduling algorithms in mobile ad hoc networks," ACM SIGMOBILE Mobile Computing and Communications Review, vol. 6, no. 3, pp. 36-49, 2002.

[8] S. Chen and K. Nahrsted, "Distributed Quality of Service Routing in Adhoc Networks," IEEE JSAC 17(8): 1488-1505, Aug 1999.

[9] Royer E. M. and Chai Keong Toh," A Review of current Routing protocols for Ad-hoc Networks", IEEE Personol Commut,. Vol. 6, No. 2, pp 46-55, April 1999

[10] Shigang Chen and Klnn Nahtstedt, "Disltibuted Quality of Service routing in Ad hoc netwoks', IEEE journal on selected area in Commu., Vol. 17., No. 8, pp 1458-1504, August 1999.

[11] David B. Johnson"Routing in Ad-hoc networks of mobile hosts" Proc of IEEE workshop on Mobile Computing System and Application, pp 1-5 Dec. 1994.

[12] http://www.computing.surrey.ac.uk/courses/cs364/FuzzyLogicFuzzySystems 3.ppt. 